\title{
Catálogo de Apoidea da Região Neotropical (Hymenoptera, Colletidae). IV. Hylaeinae ${ }^{1}$
}

\author{
Danúncia Urban ${ }^{2}$ \\ Jesus Santiago Moure ${ }^{2}$
}

\begin{abstract}
Catalogue of the apoidea of the Neotropical region (Hymenoptera, Colletidae) IV. Hylaeinae. The type localities, depositary museums, taxonomical comments and host plants of the species of Hylaeus Fabricius, 1793, the unique genus of the Hylaeinae in the Neotropical Region, are mentioned. Lectotypes of the following species are designated: Prosopis bothros Schrottky, 1910; Prosopis fissa Vachal, 1901; Prosopis itapuensis Schrottky, 1906; Prosopis opaca Schrottky, 1906; Prosopis opaciventris Friese, 1925; Prosopis paraguayensis Schrottky, 1906; Prosopis recisa Vachal, 1910; Prosopis scabra Vachal, 1909; Prosopis sculptilis Schrottky, 1910; Prosopis tricolor Schrottky, 1906 and Prosopis trivittata Friese, 1916. The following ones are recognized as new combinations: Hylaeus petroselini (Schrottky, 1906); Hylaeus polybioides (Schrottky, 1906), Hylaeus polybiaeformis (Schrottky, 1907) and Hylaeus psammophilus (Schrottky, 1906).
\end{abstract}

KEY WORDS. Hymenoptera, Colletidae, Hylaeinae, Hylaeus, Neotropical

Os Hylaeinae são representados na região Neotropical por Hylaeus, um gênero de abelhas pequenas e graciosas, geralmente com ornamentação amarela na cabeça e mesosoma e a língua truncada ou fracamente bífida. VACHAL $(1901,1909,1910)$ deu a conhecer numerosas espécies sulamericanas de Hylaeus publicadas no gênero Prosopis Fabricius, 1804, sendo que as de 1909 e 1910 não têm uma descrição formal, aparecem somente na chave para a identificação das espécies. MOURE (1960) examinou e redescreveu as espécies de Vachal, propondo chaves para a sua identificação. Para as espécies neotropicais de Hylaeus foram propostos os sub-gêneros: Hylaeana e Hylaeopsis por MichENER (1954), Cephylaeus por MOURE (1972), Gongyloprosopis por SNELLING (1982) e Orohylaeus por MiCHENER (2000b). TORO et al. (1989) constataram uma espécie do sub-gênero paleártico Spatulariella Popov, 1939, que foi introduzida no Chile; e, duas espécies neárticas do sub-gênero Prosopis, citadas por SNELLING (1966 e 1982), que também foram coletadas em Veracruz, México.

$\mathrm{Na}$ elaboração deste trabalho as citações bibliográficas mais freqüentes foram listadas nas "Referências Bibliográficas" e quando repetidas em uma mesma espécie foram usadas abreviadamente, as novas procedências foram obtidas nos autores consultados e as abreviações latinas são as comumente utilizadas em taxonomia. Os subgêneros foram mencionados somente quando citados pelos autores, isto porque algumas espécies são conhecidas apenas pela descrição original e as reestudadas não tiveram este enfoque.

1) Contribuição número 1296 do Departamento de Zoologia, Universidade Federal do Paraná.

2) Departamento de Zoologia, Universidade Federal do Paraná. Caixa Postal 19020, 81531-990 Curitiba, Paraná, Brasil. Bolsista do CNPq.

Revta bras. Zool. 19 (1): 31 - 56, 2002 
Os acrônimos dos museus citados no texto são relacionados a seguir: (AMNH) American Museum of Natural History, New York, Estados Unidos; (ANSP) Academy of Natural Sciences, Philadelphia, Estados Unidos; (BMNH) The Natural History Museum, London, Inglaterra; (CAS) California Academy of Sciences, San Francisco, Estados Unidos; (DZUP) Coleção Pe. J.S. Moure, Departamento de Zoologia da Universidade Federal do Paraná, Curitiba, Brasil; (LACM) Los Angeles County Museum of Natural History, Los Angeles, California, Estados Unidos; (MCZ) Museum of Comparative Zoology, Harvard University, Cambridge, Estados Unidos; (MNHP) Muséum National d'Histoire Naturelle, Paris, França; (MNHU) Museum für Naturkunde der Humboldt-Universität zu Berlin, Alemanha; (MZSP) Museu de Zoologia, Universidade de São Paulo, Brasil (ex - Museu Paulista); (SEMK) Snow Entomological Museum, University of Kansas, Lawrence, Estados Unidos; (USNM) National Museum of Natural History, Washington, Estados Unidos. O material-tipo das espécies descritas por Schrottky, em parte, foi examinado, quanto ao restante, não foi constatado nas coleções examinadas por J.S. Moure.

\section{CONTEÚDO}

Introdução.................................... 31 Hylaeus (Hylaeopsis) .................... 48

Hylaeus....................................... 32 Hylaeus (Orohylaeus) ................... 52

Hylaeus (Cephylaeus) ....................45 Hylaeus (Prosopis)......................... 52

Hylaeus (Gongyloprosopis) ............45 Hylaeus (Spatulariella).................. 53

Hylaeus (Hylaeana)....................... 46 Índice........................................... 54

\section{Hylaeus Fabricius, 1793}

Hylaeus Fabricius, 1793. Ent. Syst. Emendata et Aucta 2: 302. Espécie-tipo: Prosopis annulata Fabricius, 1804 [= Apis annulata Linnaeus, 1758], por desigação de Latreille, 1810: 438.

Prosopis Fabricius 1804. Systema Piezatorum: 293. Espécie-tipo: Mellinus bipunctatus Fabricius, 1798 [= Sphex signata Panzer, 1798], designada por Morice \& Durrant, 1915: 416.

Taxonomia. Michener, 2000a: 182-183 (comentou a prioridade de Hylaeus sobre Prosopis, que é válido como sub-gênero holártico). - Snelling in Michener, 2000a: 189-191 (propôs uma chave para os sugêneros de Hylaeus do Hemisfério Ocidental).

alampes Moure. BRASIL, São Paulo, São Paulo (localidade-tipo); Mato Grosso, Salobra.

Hylaeus alampes Moure, 1942. Papéis Avulsos, Departamento de Zoologia, São Paulo, II (21): 293. Holótipo fêmea e parátipo fêmea no DZUP.

amazonicus (Gribodo). Peru, Loreto, Pebas, Alto Amazonas (localidade-tipo), Baixo Marañon.

Prosopis amazonica Gribodo, 1894. Bol. Soc. Ent. Ital.: 294. Síntipos macho e fêmea na Coleção Gribodo, no Museu Civico di Storia Naturale, Gênova.

Hylaeus amazonicus; Meade-Waldo, 1923: 31. 
Taxonomia. Moure, 1960: 14 (redescreveu a fêmea como holótipo e o macho como alótipo).

arsenicus (Vachal). BRASIL, Goiás (localidade-tipo).

Prosopis arsenica Vachal, 1901. Ann. Soc. Ent. France 70: 81. Holótipo fêmea na coleção Vachal, MNHP.

Hylaeus arsenicus; Cockerell, 1907. Smiths. Misc. Coll. 48: 268.

Taxonomia. Moure, 1960: 62 (redescreveu o holótipo e comparou com $H$. fossisternis (Vachal, 1909) $[=$ H. bertonii $]$ ).

asper (Vachal). BolíviA, Mapiri (localidade-tipo).

Prosopis aspera Vachal, 1909. Rev. d'Ent., Caen, 28: 61. Holótipo macho na coleção Vachal, MNHP.

Hylaeus asperus; Meade-Waldo, 1923: 31.

Hylaeus asper; Moure, 1960: 39.

Taxonomia. Moure, 1960: 39 (redescreveu o holótipo macho).

aspricollis (Vachal). PERU, Callanga (localidade-tipo). GUIANA.

Prosopis aspricollis Vachal, 1901. Ann. Soc. Ent. France 70: 80. Holótipo fêmea na coleção Vachal, MNHP.

Hylaeus aspricollis; Meade-Waldo, 1923: 31.

Taxonomia. Vachal, 1901: 80 (estudou apenas uma fêmea e comentou sua semelhança com Hylaeus scrobicaudae [sic] (Vachal, 1901)). - Vachal, 1910: 66 (ocorrência na Guiana). - Moure, 1960: 63 (designou como lectótipo a fêmea de Callanga e examinou um exemplar da Guiana).

atripes (Vachal). PERU, Callanga (localidade-tipo).

Prosopis atripes Vachal, 1901. Ann. Soc. Ent. France 70: 82. Holótipo fêmea na coleção Vachal, MNHP.

Hylaeus atripes; Meade-Waldo, 1923: 31.

Taxonomia. Moure, 1960: 80 (redescreveu o holótipo).

basirufus (Vachal). BOLÍvIA, Mapiri (localidade-tipo).

Prosopis basirufa Vachal, 1910. Rev. d'Ent., Caen, 28: 67. Holótipo fêmea na coleção Vachal, MNHP.

Hylaeus basirufus; Meade-Waldo, 1923: 31.

Taxonomia. Moure, 1960: 22 (redescreveu o holótipo).

bequaerti (Schrottky). PERU, Chanchamayo (localidade-tipo) 700-1000m.

Prosopis Bequaerti [sic] Schrottky, 1910. Wien Ent. Zeitung 39 (IV): 134. Holótipo fêmea. Desconhecido o depositário.

Hylaeus bequaerti; Meade-Waldo, 1923: 31.

bertonii (Schrottky). PARAGUAI, Puerto Bertoni (localidade-tipo). BolívIA, Mapiri (localidade-tipo de P. fossisternis).

Prosopis bertonii Schrottky, 1907. An. Ci. Paraguayos 7 (1): 78 . Vários síntipos fêmeas. Desconhecido o depositário. 
Prosopis fossisternis Vachal, 1909. Rev. d'Ent., Caen, 28: 60. Holótipo fêmea na coleção Vachal, MNHP. Moure, 1960: 38; syn.

Hylaeus bertoni [sic]; Meade-Waldo, 1923: 31.

Hylaeus fossisternis; Meade-Waldo, 1923: 32.

Taxonomia. Moure, 1960: 38 (redescreveu o tipo de $P$. fossisternis e comentou o engano de Vachal ao citá-lo como macho).

binus (Vachal). BolíviA, Mapiri (localidade-tipo).

Prosopis bina Vachal, 1909. Rev. d'Ent., Caen, 28: 63. Holótipo fêmea na coleção Vachal, MNHP.

Hylaeus binus; Meade-Waldo, 1923: 31.

Taxonomia. Vachal, 1909: 63 (não indicou a procedência do exemplar). Vachal, 1910: 66 (citou novamente a fêmea na chave para identificação). Moure, 1960: 69 (redescreveu o holótipo e citou a localidade-tipo).

biscutellus (Vachal). BoLÍVIA, Mapiri (localidade-tipo).

Prosopis biscutella Vachal, 1909. Rev. d'Ent., Caen, 28: 64. Holótipo fêmea na coleção Vachal, MNHP.

Hylaeus biscutellus; Meade-Waldo, 1923: 31.

Taxonomia. Moure, 1960: 51 (redescreveu o holótipo).

bolivianus (Schrottky). BolíviA, La Paz, Mapiri (localidade-tipo).

Prosopis boliviana Schrottky, 1910. Wien Ent. Zeitung 39 (IV): 136. Treze síntipos fêmeas, sem indicação do local onde foram depositados.

Hylaeus bolivianus; Meade-Waldo, 1923: 31.

Taxonomia. Schrottky, 1910: 136 (comparou com Prosopis palmaris Vachal, 1901 e Prosopis aspricollis Vachal, 1901).

bothros (Schrottky). BolíviA, La Paz, Mapiri (localidade-tipo).

Prosopis bothros Schrottky, 1910. Wien Ent. Zeitung 39 (IV): 137. Lectótipo fêmea no AMNH, aqui designado; examinado por J.S. Moure.

Hylaeus bothros; Meade-Waldo, 1923: 31.

Taxonomia. Schrottky, 1910: 137 (examinou 21 exemplares porém não indicou o local onde foi depositado o material-tipo, comparou com Prosopis paradoxa Schrottky, 1907).

brachyceratomerus (Moure). BRASIL, São Paulo, Lussanvira (localidade-tipo).

Prosopis brachyceratomera Moure, 1941. Arq. Mus. Paulista I: 41. Holótipo macho no DZUP.

Hylaeus brachyceratomera Moure, 1942. Papéis Avulsos, Departamento de Zoologia, São Paulo, II (21): 295.

brasiliensis (Schrottky). BRASIL, São Paulo, Piracicaba (localidade-tipo).

Prosopis brasiliensis Schrottky, 1910. Wien Ent. Zeitung 39 (IV): 134.

Síntipo macho depositado no MZSP, antigo Museu Paulista, coletado em XI. 1906.

Hylaeus brasiliensis; Meade-Waldo, 1923: 31. 
Taxonomia. Schrottky, 1910: 134 (ao descrever a espécie comparou com Prosopis paraguayensis Schrottky, 1906).

breviradius (Vachal). BolíviA (localidade-tipo), Mapiri.

Prosopis breviradia Vachal, 1901. Ann. Soc. Ent. France 70: 80. Holótipo fêmea na coleção Vachal, MNHP.

Hylaeus breviradius; Meade-Waldo, 1923: 31.

Taxonomia. Vachal, 1901: 81 (citou uma fêmea da Bolívia ao descrever a espécie). - Vachal, 1909: 63 (na chave para as espécies de Prosopis citou duas fêmeas de Mapiri). - Moure, 1960: 78 (redescreveu a fêmea e referiu-se ao exemplar de Mapiri como lectótipo e à fêmea com etiqueta de Bolívia, como parátipo).

caarendyensis (Schrottky). PARAGUAI, Alto Paraná, Puerto Caarendy (localidadetipo).

Prosopis caarendyensis Schrottky, 1906. An. Ci. Paraguayos 6 (1): 23. Dois síntipos machos. Desconhecido o depositário.

Hylaeus caarendyensis; Meade-Waldo, 1923: 31.

cockerelli (Schrottky). PARAGUAI, Villa Encarnación (localidade-tipo).

Prosopis cockerelli Schrottky, 1906. An. Ci. Paraguayos 6 (1): 2. Síntipo macho coletado em 4.I.[19]05. Depositário desconhecido.

Hylaeus cockerelli; Meade-Waldo, 1923: 31.

Taxonomia. Schrottky, 1907: 270 (citou como espécie nova, redescreveu o macho).

Biologia. Schrottky, 1906: 21 (em flores de "perejil" [Petroselinum crispum (Miller) A. W. Hill]).

coroicensis (Cockerell). BolíviA, Yungas, Coroico (localidade-tipo).

Prosopis coroicensis Cockerell, 1918. Ann. Mag. Nat. Hist. (9) 2: 422.

Holótipo macho coletado em V. 1899, depositado no USNM, com o ${ }^{\circ}$

23.208; examinado por J.S. Moure.

Hylaeus corvicensis [sic]; Meade-Waldo, 1923: 31.

Taxonomia. Cockerell, 1918: 422 (comparou com Prosopis petroselini Schrottky, 1906).

cribellatus (Vachal). PERU, Callanga (localidade-tipo).

Prosopis cribellata Vachal, 1901. Ann. Soc. Ent. France 70: 82. Holótipo macho na coleção Vachal, MNHP.

Hylaeus cribellatus; Meade-Waldo, 1923: 31.

Taxonomia. Vachal, 1901: 82 (comentou que poderia ser o macho de Prosopis arsenica). - Moure, 1960: 41 (redescreveu o holótipo macho).

crustatus (Vachal). GUIANA (localidade-tipo).

Prosopis crustata Vachal, 1909. Rev. d'Ent., Caen, 28: 61. Holótipo fêmea na coleção Vachal, MNHP. 
Hylaeus crustatus; Meade-Waldo, 1923: 31.

Taxonomia. Moure, 1960: 48 (redescreveu o holótipo).

culiciformis (Schrottky). PARAGUAI, Villa Encarnación (localidade-tipo).

Prosopis culiciformis Schrottky, 1906. An. Ci. Paraguayos 6 (1): 22. Síntipos machos, coletados em 10 e 19.I.1905. Depositário desconhecido.

Hylaeus culiciformis; Meade-Waldo, 1923: 31.

Taxonomia. Schrottky, 1907: 271 (citou como espécie nova, redescreveu o macho).

Biologia. Schrottky, 1906: 22 (em flores de "perejil" [Petroselinum crispum]).

curtellus Moure. BolíviA (Mapiri (localidade-tipo).

Hylaeus curtellus Moure, 1960. Bol. Mus. Paraense E. Goeldi 27: 19. Holótipo fêmea na Coleção Vachal, MNHP.

curtulus (Vachal). Bolívia, Mapiri (localidade-tipo). PERU, Callanga.

Prosopis curtula Vachal, 1910. Rev. d'Ent., Caen, 28: 68. Lectótipo fêmea

e um paralectótipo macho na coleção Vachal, MNHP.

Hylaeus curtulus; Meade-Waldo, 1923: 31.

Taxonomia. Vachal, 1910: 68 (ao descrever a espécie citou duas fêmeas e um macho). - Moure, 1960: 28 (designou o lectótipo e redescreveu a espécie).

cuscoanus (Strand) PERU, Cuzco (localidade-tipo).

Prosopis cuscoana Strand, 1911. Arch. für Naturg. 77 (1), Suppl. 2: 156. Holótipo fêmea no MNHP.

Hylaeus cuscoanus; Meade-Waldo. 1923: 31.

Hylaeus cuzcoanus [sic]; Moure, 1960: 72.

Taxonomia. Strand, 1911: 157 (parecido com Gorytes). - Moure, 1960: 72 (redescreveu o holótipo e comentou ser próximo de H. binus Vachal, 1909).

exiguus (Schrottky). BRASIL, São Paulo, Victoria (perto de Botucatu) (localidadetipo), Piracicaba.

Prosopis exigua Schrottky, 1902. Rev. Mus. Paulista 5: 341. Síntipos machos coletados em 26.VII e 10.X, por A. Hempel. Depositados no MZSP.

Hylaeus exiguus; Meade-Waldo, 1923: 32.

Taxonomia. Schrottky, 1910: 138 (descreveu a fêmea, coletada em Piracicaba).

expansus (Vachal). PERU, Marcapata (localidade-tipo).

Prosopis expansa Vachal, 1909. Rev. d'Ent., Caen, 28: 62. Holótipo fêmea na coleção Vachal, MNHP.

Hylaeus expansus; Meade-Waldo, 1923: 32.

Taxonomia. Moure, 1960: 81(redescreveu o holótipo e comentou ser parecido com H. atripes (Vachal, 1901)). 
femoralis (Schrottky). BRASIL, São Paulo, Victoria (perto de Botucatu) (localidade-tipo). PARAGUAI, Villa Encarnación.

Prosopis femoralis Schrottky, 1902. Rev. Mus. Paulista 5: 339. Síntipos machos coletados em 26.VII.1900, depositados no MZSP.

Hylaeus femoralis; Meade-Waldo, 1923: 32.

Taxonomia. Schrottky, 1902: 339 (descreveu fêmea e macho). - Schrottky, 1906: 14 (comentou ser semelhante a $P$. rugosa Smith, 1879, como também a probabilidade de ser o macho de $P$. gracillima Schrottky, 1902). - Schrottky, 1907: 264-265 (redescreveu o macho e comentou que ambos os espécimens descritos em 1902 eram machos).

Biologia. Schrottky, 1906: 13 (em flores de "perejil" [Petroselinum crispum]).

fissus (Vachal). Bolívia (localidade-tipo), Mapiri.

Prosopis fissa Vachal, 1901. Ann. Soc. Ent. France 70: 80. Lectótipo fêmea e paralectótipo macho aqui designados, na Coleção Vachal, MNHP; examinados por J.S. Moure.

Hylaeus fissus; Meade-Waldo, 1923: 32.

Taxonomia. Vachal, 1910: 70 (colocou na chave e referiu-se à localidade de Mapiri). - Moure, 1960: 8 (redescreveu a fêmea como holótipo e o macho como alótipo, não designou lectótipo).

flavohumeralis (Cockerell). PARAGUAI, San Bernardino (localidade-tipo).

Prosopis flavohumeralis Cockerell, 1918, Ann. Mag. Nat. Hist. (9) 2: 423.

Síntipo fêmea no USNM, n. 23.209; examinado por J.S. Moure.

Hylaeus flavohumeralis; Meade-Waldo, 1923: 32.

Taxonomia. Cockerell, 1918: 423 (comparou com Prosopis itapuensis Schrottky, 1906 e Prosopis lychnis Vachal, 1910).

formosus Krombein. BAHAMAS, Bimini Island, Easter Cay (localidade-tipo).

Hylaeus formosus Krombein, 1953. Amer. Mus. Novit. 1633: 19. Síntipo fêmea no AMNH.

geminus (Vachal). BolíVIA, Mapiri (localidade-tipo).

Prosopis gemina Vachal, 1910. Rev.d'Ent., Caen, 28: 67. Lectótipo fêmea na coleção Vachal, MNHP.

Hylaeus geminus; Meade-Waldo, 1923: 32.

Taxonomia. Vachal, 1910: 67 (referiu-se a duas fêmeas). - Moure, 1960: 24 (ao redescrever a espécie designou uma das fêmeas como lectótipo de $P$. gemina e a outra descreveu como Hylaeus villosellus).

genualis (Vachal). BoLívIA, Mapiri (localidade-tipo).

Prosopis genualis Vachal, 1909. Rev. d'Ent., Caen, 28: 64. Holótipo fêmea na coleção Vachal, MNHP.

Hylaeus genualis; Meade-Waldo, 1923: 32. 
Taxonomia. Vachal, 1909: 64 (não indicou nem a localidade nem o sexo do tipo). - Moure, 1960: 50 (localizou no MNHP o holótipo fêmea de Mapiri, redescreveu-o e comparou com H. biscutellus (Vachal, 1909)).

guaraniticus (Schrottky). PARAGUAI, Villa Encarnación (localidade-tipo).

Prosopis guaranitica Schrottky, 1906. An. Ci. Paraguayos 6 (1): 10.

Síntipo macho coletado em XII. 1904 na CAS.

Hylaeus guaranticus [sic]; Meade-Waldo, 1923: 32.

Taxonomia. Schrottky, 1907: 263 (citou como espécie nova e redescreveu o macho).

Biologia. Schrottky, 1906: 10 (visitando flores de "perejil" [Petroselinum crispum]).

hydrophilus (Schrottky). PARAGUaI, Alto Paraná, Puerto Caarendy (localidadetipo).

Prosopis hydrophila Schrottky, 1906. An. Ci. Paraguayos 6 (1): 24. Oito machos síntipos, coletados em XI. [19]05; um síntipo macho na CAS, examinado por J.S. Moure e um síntipo fêmea no MLP.

Hylaeus hydrophilus; Meade-Waldo, 1923: 32.

Taxonomia. Durante \& Díaz, 1998. Rev. Mus. La Plata (N. S.) Serie Tecnica y Didactica 33: 9 (examinaram um síntipo fêmea no MLP, nº 2163 , sem o tagma cefálico).

iheringi (Schrottky). PARAGUAI, Puerto Bertoni (localidade-tipo). BRASIL.

Prosopis iheringi Schrottky, 1910. Rev. Mus. Paulista 8: 71. Síntipos:

fêmea coletada em 20.V.[19]09 e macho coletado em 13.I.[19]09.

Depositário desconhecido.

Hylaeus iheringi; Meade-Waldo, 1923: 32.

Taxonomia. Schrottky, 1913. An. Soc. Ci. Arg. 75: 235. (ocorrência no Brasil).

iridipennis (Schrottky). PARAguaI, Alto Paraná, Puerto Caarendy (localidadetipo).

Prosopis iridipennis Schrottky, 1906. An. Ci. Paraguayos 6 (1): 26. Holótipo macho coletado em 25.XI.1905.

Hylaeus iridipennis; Meade-Waldo, 1923: 32.

itapuensis (Schrottky). PARAGUAI, Villa Encarnación (localidade-tipo).

Prosopis itapuensis Schrottky, 1906. An. Ci. Paraguayos 6 (1): 19. Lectó-

tipo fêmea, coletado em 29.XII.04, aqui designado e paralectótipo

macho coletado em 16.XII.04, na CAS; examinados por J.S. Moure.

Hylaeus itapuensis; Meade-Waldo, 1923: 32.

Taxonomia. Schrottky, 1906: 19 (comparou a fêmea com P. variolosa Smith, 1853). - Schrottky, 1907: 268 (citou como espécie nova, redescreveu fêmea e macho e comparou com P. variolosa e P. palmaris Vachal, 1901).

Biologia. Schrottky, 1906: 19 (em flores de " perejil" [Petroselinum crispum]). 
joergenseni (Schrottky). ParaguaI, Alto Paraná, Puerto Caarendy (localidadetipo). BrasiL, Mato Grosso, Salobra.

Prosopis opaca Schrottky, 1906. An. Ci. Paraguayos 6 (1): 14; nom. praeoc. Lectótipo macho, coletado em 26.XI.1905, depositado na CAS, aqui designado.

Prosopis joergenseni Schrottky, 1913. An. Soc. Ci. Argentina: 235; nom. n. para Prosopis opaca Schrottky non Foerster, 1871.

Hylaeus opacus; Meade-Waldo, 1923: 32.

Hylaeus joergenseni; Moure, 1942. Papéis Avulsos, Departamento de Zoologia, São Paulo, II (21): 292.

Taxonomia. Moure, 1942: 292 (redescreveu o macho).

limbifrons (Cresson). CUBA (localidade-tipo).

Prosopis limbifrons Cresson, 1869. Proc. Boston Soc. Nat. Hist. 12: 273.

Síntipo fêmea na Coleção Gundlach, Museu do Instituto de Segunda Enseñanza de La Habana, CUBA.

Hylaeus limbifrons; Meade-Waldo, 1923: 32.

lumbellus (Vachal). BolíviA, Mapiri (localidade-tipo).

Prosopis lumbella Vachal, 1910. Rev. d'Ent., Caen, 28: 65. Holótipo fêmea na coleção Vachal, MNHP.

Hylaeus lumbellus; Meade-Waldo, 1923: 32.

Taxonomia. Moure, 1960: 45 (redescreveu o holótipo e comparou com $H$. biscutellus (Vachal, 1909)).

lychnis (Vachal). GUIANA (localidade-tipo).

Prosopis lychnis Vachal, 1910. Rev. d'Ent., Caen, 28: 68. Holótipo fêmea na coleção Vachal, MNHP.

Hylaeus lychnis; Meade-Waldo, 1923: 32.

Taxonomia. Moure, 1960: 27 (redescreveu o holótipo).

mapirensis (Schrottky). BolíviA, La Paz, Mapiri (localidade-tipo).

Prosopis mapirensis Schrottky, 1910. Wien Ent. Zeitung 39 (IV): 133.

Cinco síntipos fêmeas. Depositário desconhecido.

Hylaeus mapiriensis [sic]; Meade-Waldo, 1923: 32.

nasutus (Vachal). GUIANA (localidade-tipo).

Prosopis nasuta Vachal, 1910. Rev. d'Ent., Caen, 28: 67. Lectótipo fêmea e lectoparátipo fêmea na coleção Vachal, MNHP.

Hylaeus nasutus; Meade-Waldo, 1923: 32.

Taxonomia. Moure, 1960: 32 (designou e redescreveu o lectótipo).

nigripennis (Vachal). BoLÍVIA, Mapiri (localidade-tipo).

Prosopis nigripennis Vachal, 1909. Rev. d'Ent., Caen, 28: 59. Holótipo fêmea na coleção Vachal, MNHP.

Hylaeus nigripennis; Meade-Waldo, 1923: 32.

Taxonomia. Moure, 1960: 74 (redescreveu o holótipo). 
palmaris (Vachal). Bolívia (localidade-tipo).

Prosopis palmaris Vachal, 1901. Ann. Soc. Ent. France 70: 81. Holótipo macho na coleção Vachal, MNHP.

Hylaeus palmaris; Meade-Waldo, 1923: 32.

Taxonomia. Moure, 1960: 30 (redescreveu o holótipo).

pannosus (Vachal). BRASIL, Goiás (localidade-tipo), Rio Urubu.

Prosopis pannosa Vachal, 1909. Rev. d'Ent., Caen, 28: 62. Lectótipo e paralectótipo machos na Coleção Vachal, MNHP.

Hylaeus pannosus; Meade-Waldo, 1923: 32.

Taxonomia. Moure, 1960: 56 (designou e redescreveu o lectótipo, citou ocorrência no Rio Urubu e comparou com H. crustatus (Vachal, 1909)).

paradoxus (Schrottky). PARAGUAI, Puerto Bertoni (localidade-tipo).

Prosopis paradoxa Schrottky, 1907. An. Ci. Paraguayos 7 (1): 77 . Holótipo fêmea. Depositário desconhecido.

Hylaeus paradoxus; Meade-Waldo, 1923: 32.

paraguayensis (Schrottky). PARAGUAI, Villa Encarnación (localidade-tipo). ARGENTINA, Catamarca.

Prosopis paraguayensis Schrottky, 1906. An. Ci. Paraguayos 6 (1): 20.

Lectótipo macho coletado em 15.II.[19]05 aqui designado, depositado na CAS; examinado por J.S. Moure.

Hylaeus paraguayensis; Meade-Waldo, 1923: 32.

Taxonomia. Schrottky, 1906: 20-21(descreveu fêmea e macho, comentou ter depositado uma fêmea no MLP e, que esta espécie pertencia ao grupo $P$. amazonica Gribodo, 1894). - Schrottky, 1907: 269 (citou como espécie nova, redescreveu fêmea e macho). - Schrottky, 1913. An. Soc. Ci. Arg. 75: 235 (ocorrência na ARGENTINA).

paulistanus (Schrottky). BrasiL, São Paulo (localidade-tipo); Mato Grosso, Salobra. Paraguai, Alto Paraná, Puerto Caarendy.

Prosopis paulistana Schrottky, 1906. An. Ci. Paraguayos 6 (1): 12. Sínti-

pos: macho coletado em 10.X.1901 (São Paulo) e três machos em 26.XI.1905 (Puerto Caarendy). Depositário desconhecido.

Hylaeus paulistanus; Meade-Waldo, 1923: 32.

Taxonomia. Schrottky, 1907: 264 (citou como espécie nova e redescreveu o macho coletado em São Paulo). - Moure, 1942. Papéis Avulsos, Departamento de Zoologia, São Paulo, 2 (21): 293 (ocorrência em Salobra).

peruvianus (Schrottky). PERU, Chanchamayo (localidade-tipo).

Proposis peruviana Schrottky, 1910. Wien Ent. Zeitung 39 (IV): 135.

Holótipo macho. Desconhecido o depositário.

Hylaeus peruvianus; Meade-Waldo, 1923: 32.

Taxonomia. Schrottky, 1910: 135 (citou Prosopis cribellata Vachal, 1901, na descrição). 
petroselini (Schrottky). PARAGUAI, Villa Encarnación (localidade-tipo). comb. n. Prosopis petroselini Schrottky, 1906. An. Ci. Paraguayos 6 (1): 8. Holótipo macho, coletado em XII. 1904. Depositário desconhecido.

Taxonomia. Schrottky, 1907: 261 (citou como espécie nova e redescreveu o macho).

Biologia. Schrottky, 1906: 8 (em flores de Petroselinum sativum Hoffm. [= Petroselinum crispum (Miller) A.W. Hill]).

polybioides (Schrottky). PARAGUAI, Villa Encarnación (localidade-tipo). comb. n. Prosopis polybioides Schrottky, 1906. An. Ci. Paraguayos 6 (1): 9. Síntipo. fêmea coletado em 30.XII.1904. Depositário desconhecido.

Taxonomia. Schrottky, 1906: 9 (comentou a semelhança com Polybia scutellaris (White, 1841)). - Schrottky, 1907: 262 (citou como espécie nova e redescreveu a fêmea).

Biologia. Schrottky, 1906: 9 (em flores de "perejil" [= Petroselinum crispum]).

polybiaeformis (Schrottky). PARAGUAI, Puerto Bertoni (localidade-tipo). comb. n. Prosopis polybiaeformis Schrottky, 1907. An. Ci. Paraguayos 7 (1): 77.

Holótipo fêmea na CAS, coletado em 22.XII.04; examinado por J.S. Moure.

psammophilus (Schrottky). PARAGUAI, Puerto Caarendy (localidade-tipo). comb. n. Prosopis arenaria Schrottky, 1906. An. Ci. Paraguayos 6 (1): 27; nom. praeoc. Dois síntipos machos coletados em 26.XI.1905. Desconhecido o depositário.

Prosopis psammophila Schrottky, 1913; nom. n. para Prosopis arenaria Schrottky non Morawitz, 1876.

puerulus (Vachal). BoLÍviA, Mapiri (localidade-tipo).

Prosopis puerula Vachal, 1910. Rev. d'Ent., Caen, 28: 70. Holótipo macho na coleção Vachal, MNHP.

Hylaeus puerulus; Meade-Waldo, 1923: 32.

Hylaeus merulus [sic]; Meade-Waldo, 1923: 32.

Taxonomia. Moure, 1960: 10 (redescreveu o holótipo).

recisus (Vachal). BolíviA, Mapiri (localidade-tipo).

Prosopis recisa Vachal, 1910. Rev. d'Ent., Caen, 28: 69. Lectótipo fêmea e paralectótipo macho aqui designados, na coleção Vachal, MNHP; examinados por J.S. Moure.

Hylaeus recisus; Meade-Waldo, 1923: 32.

Taxonomia. Moure, 1960: 12 (redescreveu a fêmea como holótipo e o macho como alótipo, não designou lectótipo).

rivalis (Schrottky). PARAGUAI, Villa Encarnación (localidade-tipo). BRASIL, Paraná, Curitiba, São José dos Pinhais. 
Prosopis rivalis Schrottky, 1906. An. Ci. Paraguayos 6 (1): 15. Síntipo fêmea coletado em 25.I.1905. Depositário desconhecido. Hylaeus rivalis; Meade-Waldo, 1923: 32.

Taxonomia. Schrottky, 1907: 267 (citou como espécie nova e redescreveu a fêmea). - Sakagami \& Laroca, 1971. Kontiû 39 (3): 219 (ocorrência em São José dos Pinhais e Curitiba).

Biologia. Schrottky, 1906: 16 (em flores de "perejil" [= Petroselinum crispum]). - Sakagami, Laroca \& Moure, 1967. Jour. Fac. Sci. Hokkaido Univ. VI Zool. 16 (2): 282 (em flores de Jussiacea sericea Camb. [= Ludwigia sericea (Camb.) Hara]).

rufulus (Friese). ARGENTINA, Tucumán (localidade-tipo).

Prosopis rufula Friese, 1908. Flora og Fauna 10: 5. Síntipo fêmea provavelmente no MNHU.

Hylaeus rufulus; Meade-Waldo, 1923: 32.

rugosus (Smith) BRASIL, Amazonas, São Paulo de Olivença. (localidade-tipo).

Prosopis rugosa Smith, 1879. Descr. N. Sp. Br. Mus. p. 22. Holótipo macho no BMNH.

Hylaeus rugosus; Meade-Waldo, 1923: 33.

Taxonomia. Moure, 1960: 55 (redescreveu o holótipo).

saltensis (Friese). ARGENTINA, Salta (localidade-tipo).

Prosopis saltensis Friese, 1908. Flora og Fauna 10: 5. Um síntipo fêmea no AMNH, coletado por Steinbach em III. [19]05; demais síntipos fêmeas provavelmente no MNHU.

Prosopis saltensis var. rufifrons Friese, 1908. Flora og Fauna 10: 5. Três síntipos fêmeas provavelmente no MNHU.

Hylaeus saltensis; Meade-Waldo, 1923: 33.

scrobicauda (Vachal). BoLÍVIA (localidade-tipo).

Prosopis scrobicauda Vachal, 1901. Ann. Soc. Ent. France 70: 79. Holótipo fêmea na coleção Vachal, MNHP.

Hylaeus scrobicauda; Meade-Waldo, 1923: 33.

Taxonomia. Moure, 1960: 47 (redescreveu o holótipo).

scrupeus (Vachal). BolíviA, Mapiri (localidade-tipo).

Prosopis scrupea Vachal, 1909. Rev. d'Ent., Caen, 28: 63. Lectótipo macho e dois paralectótipos fêmeas na coleção Vachal, MNHP.

Hylaeus scrupeus; Meade-Waldo, 1923: 33.

Taxonomia. Moure, 1960: 58 (designou o lectótipo e redescreveu os tipos). sculptilis (Schrottky). Bolívia, Mapiri (localidade-tipo).

Prosopis sculptilis Schrottky, 1910. Wien Ent. Zeitung 39 (IV): 136. Lectótipo fêmea, aqui designado, depositado no AMNH; examinado por J.S. Moure. 
Hylaeus sculptilis; Meade-Waldo, 1923: 33.

Taxonomia. Schrottky, 1910: 136 (não indicou o número de exemplares nem onde foram depositados, comparou com Prosopis gracillima Schrottky, 1902).

seabrai nom. n. PARAGUAI, Villa Encarnación (localidade-tipo).

Prosopis longicornis Schrottky, 1906. An. Ci. Paraguayos 6 (1): 28; nom. praeoc. Holótipo macho coletado em 27.XII.1904. Depositário desconhecido.

Hylaeus longicornis; Meade-Waldo, 1923: 32.

Hylaeus seabrai nom. n. para Prosopis longicornis Schrottky, 1906 non Prosopis longicornis Schenk, 1853.

Taxonomia. Schrottky, 1907: 272 (publicou como espécie nova e redescreveu o macho).

Biologia. Schrottky, 1906: 28 (em flores de "peregil" [Petroselinum crispum]).

stenops (Schrottky). BRASIL, São Paulo, Piracicaba (localidade-tipo).

Prosopis stenops Schrottky, 1910. Wien Ent. Zeitung 39 (IV): 136. Holótipo macho, coletado em XI. 1906, depositado no MZSP, antigo Museu Paulista.

Hylaeus stenops; Meade-Waldo, 1923: 33.

Taxonomia. Schrottky, 1910: 136 (comparou com Prosopis peruviana Schrottky, 1910).

stilbaspis (Vachal). BoLÍVIA (Localidade-tipo).

Prosopis stilbaspis Vachal, 1901. Ann. Soc. Ent. France 70: 80. Holótipo fêmea na coleção Vachal.

Hylaeus stilbaspis; Meade-Waldo, 1923: 33.

Taxonomia. Moure, 1960: 60 (redescreveu o holótipo).

transversus (Vachal). BRASIL, Goiás (localidade-tipo).

Prosopis transversa Vachal, 1909. Rev. d'Ent., Caen, 28: 63. Holótipo fêmea na coleção Vachal, MNHP.

Hylaeus transversus; Meade-Waldo, 1923: 33.

Taxonomia. Vachal, 1910: 66 (citou novamente a fêmea na chave para a identificação). - Moure, 1960: 71 (redescreveu o holótipo).

triangulum Fabricius. "BRASILIA" [BRASIL] (localidade-tipo).

Hylaeus triangulum Fabricius, 1793. Ent. System. 2: 306. Holótipo macho no Universitets Zoologiske Museum, Copenhagem.

Taxonomia. Moure, 1960. Bol. Mus. Paraense E. Goeldi 27: 75 (redescreveu o holótipo, comentou algumas semelhanças com Sphecodes Latreille, 1804 e Bothranthidium Moure, 1947). - Moure, 1960. Studia Ent. 3 (1/4): 100 (redescreveu o holótipo). 
trisulcus (Vachal). BoLíviA, Mapiri (localidade-tipo).

Prosopis trisulca Vachal, 1910. Rev. d'Ent., Caen, 28: 66. Holótipo fêmea na coleção Vachal, MNHP.

Hylaeus trisulcus; Meade-Waldo, 1923: 33.

Taxonomia. Moure, 1960: 20 (redescreveu o holótipo).

ubertus (Vachal). BoLÍVIA, Mapiri (localidade-tipo).

Prosopis uberta Vachal, 1910. Rev. d'Ent., Caen, 28: 65. Holótipo fêmea na coleção Vachal, MNHP.

Hylaeus ubertus; Meade-Waldo, 1923: 33.

Taxonomia. Vachal, 1910: 65 (citou um macho de Mapiri). - Moure, 1960:

36 (comentou que o holótipo era uma fêmea, e não macho, e o redescreveu).

vachali Meade-Waldo. BolíviA, Mapiri (localidade-tipo). PERU, Pachitea.

Prosopis scabra Vachal, 1909. Rev. d'Ent., Caen, 28: 62 [macho], 64 [fêmea]; nom. praeoc. Lectótipo macho na coleção Vachal, MNHP.

Hylaeus vachali Meade-Waldo, 1923: 33; nom. n. para P. scabra Vachal non Fabricius, 1798).

Taxonomia. Vachal, 1909: 62 e 64 (citou um macho de Mapiri (62) e uma fêmea também de Mapiri (64)). - Moure, 1960: 42 (designou como lectótipo um macho de Pachitea, Peru, comentando que Vachal registrou o macho como sendo de Mapiri, Bolívia; e descreveu a fêmea de P. scabra como Hylaeus vachalianus).

vachalianus Moure. BoLíviA, Mapiri (localidade-tipo).

Hylaeus vachalianus Moure, 1960. Bol. Mus. Paraense E. Goeldi 27: 44. Holótipo fêmea no MNHP.

variolosus (Smith). BRASIL, Pará, Santarém (localidade-tipo).

Prosopis variolosa Smith, 1853. Cat. Hym. Br. Mus. 1 p. 24. Holótipo macho no BMNH.

Hylaeus variolosus; Meade-Waldo, 1923: 33.

Taxonomia. Smith, 1853: 24 (descreveu como fêmea). - Moure, 1960: 77 (redescreveu o holótipo macho que Smith havia identificado como fêmea).

villosellus Moure. BolíviA, Mapiri (localidade-tipo).

Hylaeus villosellus Moure, 1960. Bol. Mus. Paraense E. Goeldi 27: 25. Holótipo fêmea no MNHP.

xanthocephalus (Schrottky). PARAGUAI, Villa Encarnación (localidade-tipo).

Prosopis xanthocephala Schrottky, 1906. An. Ci. Paraguayos 6 (1): 25.

Síntipos machos coletados em 29.XII.1904. Depositário desconhecido. Hylaeus xanthocephalus; Meade-Waldo, 1923: 33.

Taxonomia. Schrottky, 1907: 272 (citou como espécie nova e redescreveu o macho).

Biologia. Schrottky, 1906: 26 (flores de "peregil” [Petroselinum crispum]). 
yaguarae (Schrottky). PARAGUAI, Villa Encarnación (localidade-tipo).

Prosopis tristis Schrottky, 1906. An. Ci. Paraguayos 6 (1): 24; nom.

praeoc. Síntipos machos coletados em 22.XII.1904. Depositário desconhecido.

Prosopis yaguarae Schrottky, 1913. An. Soc. Ci. Arg. 75: 235; nom. n. para

Prosopis tristis non Frey-Gessner,1900.

Hylaeus tristissimus Meade-Waldo, 1923: 33; nom. n. para Prosopis tristis

Schrottky non Frey-Gessner, 1900.

Taxonomia. Schrottky, 1907: 271(citou P. tristis como espécie nova, redescreveu o macho).

Biologia. Schrottky, 1906: 24 (flores de "peregil" [Petroselinum crispum]).

\section{Hylaeus (Cephylaeus) Moure, 1972}

Hylaeus (Cephylaeus) Moure, 1972. Rev. Brasil. Biol. 32 (2): 280. Espécie-tipo: Hylaeus (Cephylaeus) larocai Moure, 1972. Designação original.

larocai Moure. BRASIL, Paraná, Curitiba (localidade-tipo).

Hylaeus (Cephylaeus) larocai Moure, 1972. Rev. Brasil. Biol. 32. (2): 281. Holótipo macho e alótipo fêmea no DZUP.

\section{Hylaeus (Gongyloprosopis) Snelling, 1982}

Hylaeus (Gongyloprosopis) Snelling, 1982. Bull. So. California Acad. Sci. 81 (1): 16. Espécie-tipo: Prosopis cruenta Vachal, 1910. Designação original.

cruentus (Vachal). Guiana (localidade-tipo). Guiana Francesa, Ile Royal, Iles du Salut.

Prosopis cruenta Vachal, 1910. Rev. d'Ent., Caen, 28: 69. Holótipo fêmea na coleção Vachal, MNHP.

Hylaeus cruentus; Meade-Waldo, 1923: 31.

Hylaeus (Gongyloprosopis) cruentus; Snelling, 1982: 18.

Taxonomia. Moure, 1960: 7 (redescreveu o holótipo fêmea). - Snelling, 1982: 18-21(redescreveu a fêmea, comparou com H. orbicus (Vachal, 1910) e H. preposterosus Snelling, 1982).

orbicus (Vachal). BRITISH GUIANA [GUIANA] (localidade-tipo). GUIANA FRANCESA, Ile Royal, Iles du Salut.

Prosopis orbica Vachal, 1910. Rev. d'Ent., Caen, 28: 69. Holótipo macho na coleção Vachal, MNHP.

Hylaeus orbicus; Meade-Waldo, 1923: 32.

Hylaeus (Gongyloprosopis) orbicus; Snelling, 1982: 21.

Taxonomia. Vachal, 1910: 69 (não indicou a localidade-tipo). - Moure, 1960: 6 (anotou "British Guiana" como localidade do tipo, redescreveu o holótipo e comparou com H. cruentus (Vachal, 1910)). 
preposterosus Snelling. BolíviA, Beni, $20 \mathrm{~km} \mathrm{~W} \mathrm{Laranjeiras} \mathrm{(localidade-tipo);}$ Beni, Rio Itenez, Pampa do Meio e Foz do Rio Baures.

Hylaeus (Gongyloprosopis) preposterosus Snelling, 1982. Bull. So. California Acad. Sci. 81 (1): 21. Holótipo macho e alótipo no AMNH, parátipo fêmea no LACM.

Taxonomia. Snelling, 1982: 23 (comparou com H. cruentus e H. orbicus e elaborou uma chave para identificar as três espécies).

\section{Hylaeus (Hylaeana) Michener, 1954}

Hylaeus (Hylaeana) Michener, 1954. Bull. Amer. Mus. Nat. Hist. 104: 28. Espécie-tipo: Hylaeus panamensis Michener, 1954. Designação original.

costaricensis (Friese). Costa RicA, San José (localidade-tipo).

Prosopis costaricensis Friese, 1916. Stett. ent. Ztg. 77: 301. Três síntipos machos provavelmente no MNHU.

Hylaeus costaricensis; Meade-Waldo, 1923: 31.

Hylaeus (Hylaeana) costaricensis; Snelling, 1982: 4.

Taxonomia. Friese, 1916: 301 (comparou com P. rufoclypeata Friese descrita no mesmo trabalho).

dictyotus Snelling. GuiANa FrancESA, Ile Royal, Iles du Salut (localidade-tipo);

14 km SE Sinnamary, Kourou, Montagne de Pere. TRINIDAD, Cumuto.

Hylaeus (Hylaeana) dictyotus Snelling, 1982. Bull. So. California Acad.

Sci 81 (1): 13. Holótipo macho, alótipo, 19 machos e 13 fêmeas parátipos. Holótipo, alótipo e maioria dos parátipos no SEMK, cinco machos e quatro fêmeas parátipos no LACM.

Taxonomia. Snelling, 1982: 16 (comparou com H. cruentus (Vachal, 1910), H. rawi Snelling, 1982, e H. extrinsecus Snelling, 1982).

extrinsecus Snelling. JAMAICA, Balaclava (localidade-tipo).

Hylaeus (Hylaeana) extrinsecus Snelling, 1982. Bull. So. California Acad.

Sci. 81 (1): 11. Holótipo fêmea no MCZ.

Taxonomia. Snelling, 1982: 13 (comentou que poderia ser a fêmea de $H$. phaeoscapus Snelling, 1982).

knabi (Cockerell) GuAtemala, Champerico (localidade-tipo). MÉXICO.

Prosopis knabi Cockerell, 1918. Ann. Mag. Nat. Hist. (9) 2: 425. Síntipo macho no USNM, coletado em 4. VIII. [19]05; examinado por J.S. Moure.

Hylaeus knabi; Meade-Waldo, 1923: 32.

Hylaeus (Hylaeana) knabi; Snelling, 1982: 4.

Taxonomia. Snelling, 1982: 4 (ocorrência no México).

panamensis Michener. PANAMÁ, Coclé, El Valle de Antón (localidade-tipo). AMÉRICA CENTRAL.

Hylaeus (Hylaeana) panamensis Michener, 1954. Bull. Amer. Mus. Nat. 
Hist., 104: 28. Holótipo macho no AMNH e quatro parátipos machos no SEMK.

Taxonomia. Snelling, 1975. Contrib. Sci. Natur. Hist. Mus. Los Angeles 267: 8 (ocorrência até o sul do Texas, Arizona e California). - Snelling, 1982: 4 (esclareceu que esta espécie foi citada por erro como Hylaeus (Hylaeana) aztecus em sua publicação de 1968: 4).

Biologia. Krombein et al., 1979. Catalog of Hymenoptera in America North of Mexico II: 1766 (visitando flores de Dicraurus Hook. f., Hyptis emoryi Torreyi, Verbesina encelioides Benth et Hook.).

phaeoscapus Snelling. JAmAICA, Irish Town, St. Andrew (localidade-tipo).

Hylaeus (Hylaeana) phaeoscapus Snelling, 1982. Bull. So. California Acad. Sci. 81 (1): 9 . Holótipo macho no LACM.

Hylaeus (Hylaeana) phaeoscopus [sic]; Raw, 1984. Revta. Bras. Ent. 28 (4): 492.

quadratifer (Cockerell). GUATEMALA Gualán (localidade-tipo).

Prosopis quadratifera Cockerell, 1912. Ann. Mag. Nat. Hist. (8) 9: 566.

Síntipo macho. Depositário desconhecido.

Hylaeus (Hylaeana) quadratiferus; Snelling, 1982: 4.

Biologia. Cockerell, 1912. Can. Ent. 44: 281 (em flores de Iresine paniculata (L.) [= Iresine diffusa H. \& B. ex Willd.]).

rawi Snelling. JAMAICA, Port Royal, St. Andrew (localidade-tipo).

Hylaeus (Hylaeana) rawi Snelling, 1982. Bull. So. California Acad. Sci.

81 (1): 5. Holótipo, alótipo e um parátipo no LACM, um parátipo na Coleção A. W. Raw.

royesi Raw. JAMAICA, St. Catherine, $3 \mathrm{~km}$ ao norte de Hellshire Point (localidadetipo).

Hylaeus royesi Raw, 1984. Revta bras. Ent. 28 (4): 491. Holótipo na coleção do autor. Parátipos no DZUP, BMNH, USNM, LACM, e University Museum, Oxford University, Oxford.

Taxonomia. Raw, 1984: 492 (comparou com as espécies jamaicanas de Hylaeus: H. extrinsecus Snelling, 1982, H. phaeoscapus Snelling, 1982; H. rawi Snelling, 1982 e H. royesi Snelling, 1984).

Biologia. Raw, 1984: 492 (visitando flores de Mikania micracantha Kunth. (Compositae) $[=$ Asteraceae $]$ ).

rufoclypeatus (Friese). CosTA RICA, San José (localidade-tipo). HonduRAs, Zamorano.

Prosopis rufoclypeata Friese, 1916. Stett. ent. Ztg. 77: 301. Um macho e duas fêmeas síntipos provavelmente no MNHU.

Hylaeus rufoclypeatus; Meade-Waldo, 1923: 32.

Hylaeus (Hylaeana) rufoclypeatus; Snelling, 1982: 5.

Taxonomia. Cockerell, 1949: 433 (ocorrência em Zamorano). 
trivittatus (Friese). CosTA RICA, San José (localidade-tipo).

Prosopis trivittata Friese, 1916. Stett. ent. Ztg. 77: 301. Lectótipo fêmea, coletado em 1913, aqui designado, depositado no AMNH; examinado . por J.S. Moure.

Hylaeus trivittatus; Meade-Waldo, 1923: 33.

Hylaeus (Hylaeana) trivittatus; Snelling, 1982: 5.

Taxonomia. Friese, 1916: 301 (não indicou número de exemplares nem depositário, comparou com P. rufoclypeata (Friese, 1916)).

\section{Hylaeus (Hylaeopsis) Michener, 1954}

Hylaeus (Hylaeopsis) Michener, 1954. Bull. Amer. Mus. Nat. Hist. 104: 27. Espécie-tipo: Prosopis mexicana Cresson, 1869. Designação original.

callosulus Meade-Waldo. Costa RiCA, San José (localidade-tipo).

Prosopis callosa Friese, 1921. Stett. ent. Ztg. 82: 82; nom. praeoc. Síntipo fêmea provavelmente no MNHU.

Hylaeus callosulus Meado-Waldo, 1923: 31; nom. n. para Prosopis callosa Friese non Cockerell, 1910.

Prosopis monacha Warncke, 1970. Rech. Agron. Gembloux Bull. (n.s.) 5:

747; nom. n. para P. callosa Friese non Cockerell, 1910. - Snelling, 1982: 3 ; syn.

Hylaeus (Hylaeopsis) callosulus; Snelling, 1982: 3.

Taxonomia. Friese, 1921: 82 (comparou com P. ruficollis Friese, 1921).

cecidonastes Moure. BRASIL, Paraná, Piraquara (localidade-tipo).

Hylaeus (Hylaeopsis) cecidonastes Moure, 1972. Rev. Brasil. Biol. 32 (2):

275. Holótipo macho, alótipo fêmea e vários parátipos machos e fêmeas no DZUP.

Taxonomia. Moure, 1972: 278 (próxima de $H$. vachali Meade-Waldo, 1923). Biologia. Moure, 1972: 275 (comentou que os exemplares de H. cecidonastes estudados nasceram em laboratório, de galhas vazias feitas pelo microlepidóptero Eucecidoses minutanus Brèthes, 1916 (Cecidoseidae)). - Laroca, 1972. Rev. Brasil. Biol. 32 (2): 285-290 (descreveu ninhos de Hylaeus cecidonastes encontrados em galhas globosas de Schinus englerii F. A. Barkley (Anacardiaceae); em geral constituidos por uma célula, feita com material semelhante a celofane e lembrando um gomo de laranja no formato; relatou o conteúdo dos ninhos estudados).

dubiosus (Cresson). MÉXICO (localidade-tipo); [Veracruz] Orizaba.

Prosopis dubiosa Cresson, 1869. Proc. Boston Soc. Nat. Hist. 12: 272. Síntipo macho na ANSP.

Hylaeus dubiosus; Meade-Waldo, 1923: 31.

Hylaeus (Hylaeopsis) dubiosus; Snelling, 1982: 3.

Taxonomia. Cockerell, 1899. Cat. Abejas de Mexico: 4 (Orizaba). 
gracillimus (Schrottky). BrasiL, São Paulo, Victoria (perto de Botucatu) (localidade-tipo). Paraguai, Villa Encarnación, Caarendy. Panamá, Coclé, El Valle de Antón.

Prosopis gracillinea Schrottky, 1902. Rev. Mus. Paulista 5: 340; laps. Dois síntipos fêmeas no MZSP, coletados em 26.VII.1900.

Prosopis gracillima; Schrottky, 1906. An. Ci. Paraguayos 6 (1): 16.

Prosopis gracillima var. paranensis Schrottky, 1906. An. Ci. Paraguayos

6 (1): 17. Síntipo fêmea. Depositário desconhecido.

Hylaeus gracillimus; Meade-Waldo, 1923: 32.

Hylaeus (Hylaeopsis) gracillimus; Michener, 1954. Bull. Amer. Mus. Nat.

Hist. 104: 27. Hylaeus (Hylaeopsis) gracillima; Snelling, 1982:4.

Taxonomia. Schrottky, 1906: 16-18 (ocorrência no Paraguai; descreveu Prosopis gracillima var. paranensis, sem indicar a localidade-tipo e afirmou que somente após o conhecimento dos machos seria possível considerar como variedade ou como espécie válida). - Schrottky, 1907: 265 (citou $P$. gracillima, considerou $P$. gracillinea como laps.); 266 (redescreveu $P$. gracillima var. paranensis de Villa Encarnación como espécie nova). Meade-Waldo, 1923: 32 (P. gracillinea como laps. cal.). - Michener, 1954: 28 (ocorrência no Panamá).

grossus (Cresson). MÉxICO (localidade-tipo); [Veracruz] Orizaba.

Prosopis grossa Cresson, 1869. Proc. Boston Soc. Nat. Hist. 12: 273. Síntipo macho na ANSP.

Prosopis maculata Friese, 1921. Stett. ent. Ztg.: 81. Dois síntipos machos, coletados 1.VII.1920; provavelmente depositados no MNHU. - Snelling, 1982. Bull. So. California Acad. Sci. 81 (1): 4; syn.

Hylaeus maculatus; Meade-Waldo, 1923: 32.

Hylaeus grossus; Meade-Waldo, 1923: 32.

Hylaeus (Hylaeopsis) grossus; Snelling, 1982: 4.

Taxonomia. Cockerell, 1899. Cat. Abejas de Mexico: 4 (Orizaba). - Friese, 1921: 81 (comparou P. maculata com P. maculipennis Smith, 1879).

gualanicus (Cockerell). GUATEMALA, Gualán (localidade-tipo). CosTA RICA, San José (localidade-tipo de P. ruficollis). HondURAS, Zamorano (localidade-tipo de $P$. albifrontella).

Prosopis gualanica Cockerell, 1912. Ann. Mag. Nat. Hist. (8) 9: 565. Síntipo macho, coletado em 18.II.1912 por W. P. Cockerell. Depositário desconhecido. - Snelling, 1982. Bull. So. California Acad. Sci. 81 (1): 4; syn.: P. ruficollis e $P$. albifrontella.

Prosopis ruficollis Friese, 1921. Stett. ent. Ztg.: 82. Seis síntipos fêmeas provavelmente depositados no MNHU. - Snelling, 1982. Bull. So. California Acad. Sci. 81 (1): 4; syn.

Prosopis albifrontella Cockerell, 1949. Proc. U.S. Nat. Mus. 98: 432. Holótipo macho depositado no USNM, com o n58.433; examinado por J.S. Moure. - Snelling, 1982: 4; syn. 
Hylaeus gualanicus; Meade-Waldo, 1923: 32.

Hylaeus ruficollis; Meade-Waldo, 1923: 32.

Hylaeus (Hylaeopsis) gualanicus; Snelling, 1982: 4.

Taxonomia. Cockerell, 1912: 566 (comparou com P. maculipennis Smith, 1879, P. mexicana Cresson, 1869 e P. paulistana Schrottky, 1906). Cockerell, 1949: 433 (comparou com P. albifrontella).

maculipennis (Smith). MÉxICO, Oajaca (localidade-tipo).

Prosopis maculipennis Smith, 1879. Descr. N. Sp. Hym. p. 23. Holótipo macho no BMNH com o número 17. A. 130; examinado por J.S. Moure.

Hylaeus maculipennis; Meade-Waldo, 1923: 32.

Hylaeus (Hylaeopsis) maculipennis; Snelling, 1982: 4.

maculosus (Friese). CosTA RICA, San José (localidade-tipo).

Prosopis trivittata var. maculosa Friese, 1921. Stett. ent. Ztg.: 81. Síntipo fêmea provavelmente no MNHU.

Hylaeus trivittatus var. maculosus; Meade-Waldo, 1923: 33.

Hylaeus (Hylaeopsis) maculosus; Snelling, 1982: 4.

Taxonomia. Friese, 1921: 81 (comparou com P. trivittata Friese, 1916).

mexicanus (Cresson). MÉxICO, [Veracruz] Orizaba (localidade-tipo), [Tamaulipas] Tampico, [Tabasco] Frontera.

Prosopis mexicana Cresson, 1869. Proc. Boston Soc. Nat. Hist. 12: 272. Síntipo fêmea na ANSP.

Hylaeus mexicana; Meade-Waldo, 1923: 32.

Hylaeus (Hylaeopsis) mexicana; Michener, 1954. Bull. Amer. Mus. Nat. Hist. 104: 27.

Taxonomia. Cockerell, 1899. Cat. Abejas de Mexico: 4 (Orizaba). - Cresson, 1916. Mem. Amer. Ent. Soc. 1: 108 (citou macho e fêmea e escolheu a fêmea como tipo). - Cockerell, 1918. Ann. Mag. Nat. Hist. (9) 2: 425 (ocorrência em Tampico e Frontera).

opaciventris (Friese). CosTA RICA, San José (localidade-tipo).

Prosopis opaciventris Friese, 1925, Stett. ent. Ztg. 86: 2. Lectótipo macho, aqui designado, depositado no AMNH; examinado por J.S. Moure. Demais síntipos provavelmente no MNHU.

Hylaeus (Hylaeopsis) opaciventris; Snelling, 1982: 4.

Taxonomia. Friese, 1925: 2 (descreveu fêmea e macho, comparou com $P$. maculata Friese [= Hylaeus grossus (Cresson, 1869)]).

subgriseus (Cockerell) MÉxICO, San Rafael, Jicoltepec (localidade-tipo), Yucatán, Chichen Itza; Veracruz.

Prosopis subgrisea Cockerell, 1918. Ann. Mag. Nat. Hist. (9) 2: 424. Síntipo fêmea no USNM.

Hylaeus subgriseus; Meade-Waldo, 1923: 33.

Hylaeus (Hylaeopsis) subgriseus; Snelling, 1982: 4. 
Taxonomia. Cockerell, 1918: 424 (comentou semelhança com P. mexicana Cresson, 1869 e P. maculipennis Smith, 1879). - Cockerell, 1932. Bull. Brooklyn Ent. Soc. 27: 10 (ocorrência).

Biologia. Cockerell, 1932. Bull. Brooklyn Ent. Soc. 27: 10 (em flores de Elvetia tinifolia L.).

tricolor (Schrottky). PARAGUAI, Villa Encarnación (localidade-tipo); San Bernardino. BRAsIL, São Paulo, Ribeirão Preto.

Prosopis tricolor Schrottky, 1906. An. Ci. Paraguayos 6 (1): 18. Lectótipo macho, coletado em 7.II.05, aqui designado, depositado na CAS; examinado por J.S. Moure.

Hylaeus tricolor; Meade-Waldo, 1923: 33.

Hylaeus (Hylaeopsis) tricolor; Sakagami \& Zucchi, 1978. Jour. Kansas Ent. Soc. 51 (4): 597.

Taxonomia. Schrottky, 1907: 268 (citou como espécie nova e redescreveu o macho), - Cockerell, 1918. Ann. Mag. Nat. Hist. (9) 2: 422 (ocorrência). Sakagami \& Zucchi, 1978: 597 (ocorrência).

Biologia. Schrottky, 1906: 18 (em flores de "perejil" [Petroselinum crispum]). - Sakagami \& Zucchi, 1978: 597-614 (descreveram ninhos encontrados em nidificações abandonadas de Trypoxylon fabricator Smith, 1873 e Mischocyttarus cassununga (R. von Ihering, 1903), com várias fêmeas, sugerindo uma vida agregada).

titanius (Friese). COSTA RICA, San José (localidade-tipo).

Prosopis titania Friese, 1925. Stett. ent. Ztg. 86: 2. Três síntipos fêmeas provavelmente no MNHU.

Hylaeus (Hylaeopsis) titanius; Snelling, 1982: 4.

Biologia. Friese, 1925: 2-3 (teceu comentários sobre dois ninhos coletados por H. Schmidt, um com 5 e outro com 13 células, em bambus com 7 a 8 $\mathrm{mm}$ de diâmetro).

vigilans (Smith) AmÉRICA CENTRAL, Val del Fuego (localidade-tipo). MÉXICO, Oaxaca (localidade-tipo de $P$. trepanda).

Prosopis vigilans Smith, 1879. Descr. N. Sp. Hym. p. 22. Holótipo fêmea no BMNH, n 17.A.129; examinado por J.S. Moure.

Prosopis trepanda Smith, 1879. Descr. N. Sp. Hym. p. 23. Holótipo fêmea no BMNH, nº 17.A.133; examinado por J.S. Moure. - Cockerell, 1904.

Can. Ent. 36: 304; syn.

Hylaeus vigilans; Meade-Waldo, 1923: 33.

Hylaeus trepandus; Meade-Waldo, 1923: 33.

Hylaeus (Hylaeopsis) vigilans: Snelling, 1982: 4.

Taxonomia. Moure, 1960: 65 (redescreveu o holótipo fêmea de $P$. vigilans e conservou $P$. trepanda na sinonímia).

zamoranicus (Cockerell). HONDURAS, Zamorano (localidade-tipo).

Prosopis zamoranica Cockerell, 1949. Proc. U.S. Nat. Mus. 98: 433. Holótipo macho depositado no USNM, n 58434. 
Hylaeus (Hylaeopsis) zamoranicus: Snelling, 1982: 4.

Taxonomia. Cockerell, 1949: 433 (comparou com P. gualanica Cockerell, 1912, e P. maculata Friese, 1921 [= Hylaeus grossus (Cresson, 1869)]).

\section{Hylaeus (Orohylaeus) Michener, 2000}

Hylaeus (Orohylaeus) Michener, 2000. Jour. Kansas Ent. Soc. 73 (1): 2. Espécie-tipo: Hylaeus benoisti Michener, 2000. Designação original.

benoisti Michener, 2000. EQUADOR, [Pichincha] Otavalo (localidade-tipo), Quito, $2850 \mathrm{~m}$.

Hylaeus (Orohylaeus) benoisti Michener, 2000. Jour. Kansas Ent. Soc. 73 (1): 4. Holótipo macho, três parátipos machos e um parátipo fêmea no MNHP; um parátipo macho no SEMK.

\section{Hylaeus (Prosopis) Fabricius, 1804}

Prosopis Fabricius, 1804: 293. Espécie-tipo: Mellinus bipunctatus Fabricius, 1798 [= Sphex signata Panzer, 1798], designada por Morice \& Durrant, 1915.

Prosapis [sic] Ashmead, 1894. Psyche 7: 293.

Taxonomia. Michener, 2000a: 203 (sub-gênero holártico com distribuição geográfica até o México: Veracruz, Durango e Baixa California). - Duas espécies neárticas deste sub-gênero, que ocorrem também em parte da região Neotropical, são incluídas e somente as localidades desta região são relacionadas.

aztecus (Cresson). MÉXICO, [Veracruz] Orizaba (localidade-tipo); Veracruz, Rio Nautla.

Prosopis azteca Cresson, 1869. Proc. Boston Soc. Nat. Hist. 12: 272. Síntipo fêmea na ANSP.

Hylaeus (Hylaeana) aztecus; Snelling, 1966. Bull. So. California Acad. Sci. 65 (3): 172.

Hylaeus (Prosopis) aztecus; Snelling, 1982: 3.

Taxonomia. Snelling, 1975. Contrib. Sci. Nat. Hist. Mus. Los Angeles 267:

8 (examinou o tipo de Prosopis azteca e concluiu que não pertencia ao sub-gênero Hylaeana).

transvittatus (Cockerell) MÉxICO [Meadow Valley] (localidade-tipo); Veracruz, Alvarado.

Prosopis transvittata Cockerell, 1917. Ann. Mag. Nat. Hist. (8) 20: 437.

Quatro fêmeas e três machos síntipos. Depositário desconhecido.

Hylaeus (Prosopis) transvittatus; Snelling, 1966. Los Angeles County

Mus. Contrib. Sci. 98: 16.

Taxonomia. Snelling, 1966: 16 (ocorrência). 


\section{Hylaeus (Spatulariella) Popov, 1939}

Prosopis (Spatulariella) Popov, 1939. Comtes Rendus (Doklady) de l'Acad. Sci. URSS (n. s.) 25: 169. Espécie-tipo: Hylaeus hyalinatus Smith, 1842. Designação original.

punctatus (Brullé). CHILE, Santiago (espécie introduzida).

Prosopis punctata Brullé, 1832. Expéd. sc. Morée. Zool. II p. 359. (EUROPA, GRÉCIA).

Hylaeus (Spatulariella) punctatus; Toro, Frederick \& Henry, 1989. Acta Ent. Chilena 15: 201.

Taxonomia. Toro, Frederick \& Henry, 1989: 201 (comentaram ser a primeira ocorrência desta espécie européia no Chile; redescreveram macho e fêmea).

Biologia. Toro, Frederick \& Henry, 1989: 201-202 (observaram muitos exemplares em flores de Alyssum saxatile L., Aloysium citriodora Orteg. ex Pers. [= Lippia citriodora H.B.K.], Lantana L. e Cichorium intybus L.; competindo pelo alimento com Apis mellifera L., 1758 e Manuelia Vachal, 1905, mariposas e sirfídeos, de março a maio).

\section{Addendum - Nomen nudum}

Prosopis patagonica Schrottky, 1913. An. Soc. Ci. Arg. 75: 235; nom. nudum.

O autor não descreveu a espécie, citou-a de Neuquen, em uma lista catalográfica de espécies da Argentina.

AGRADECIMENTOS. Ao Prof. Olavo Araujo Guimarães do Departamento de Botânica da Universidade Federal do Paraná (UFPR), pela colaboração na nomenclatura das plantas; aos Profs. Albino Morimasa Sakakibara e Gabriel Augusto R. Melo do Departamento de Zoologia da UFPR, pelas sugestões relativas à nomenclatura dos Colletini e pelo auxílio com a bibliografia.

\section{REFERÊNCIAS BIBLIOGRÁFICAS}

Meade-Waldo, G. 1923. Hymenoptera, fam. Apidae, subfam. Prosopidinae, p 1-45. In: P. Wytsman

(Ed.). Genera Insectorum 181, Brussels.

Michener, C.D. 1954. Bees of Panama. Bull. Amer. Mus. Nat. Hist. 104: 1-176.

2000a. The Bees of the World. London, John Hopkins Univ. Press. I-XIV+913p.

2000b. A High Andean Subgenus and Species of Hylaeus (Hymenoptera, Colletidae). Jour.

Kansas Ent. Soc. 73 (1): 1-5.

Moure, J.S. 1960. Os tipos das espécies neotropicais de Hylaeus da Coleção Vachal no Museu de

História Natural de Paris (Hymenoptera-Apoidea). Bol. Mus. Paraense E. Goeldi 27: 1-104.

— 1972. Descrição de duas espécies novas de Hylaeus do sul do Brasil (hymenoptera, Apoidea).

Rev. Brasil. Biol. 32 (2): 275-284.

Schrottky, C. 1906. Contribución al conocimiento de los Himenópteros del Paraguay. An. Ci.

Paraguayos $6(1): 1-32$.

- 1907. A contribution to the knowledge of some South American hymenoptera, chiefly from

Paraguay. Smith. Miscell. Coll. 48: 259-274.

(4): 133-138.

Revta bras. Zool. 19 (1): $31-56,2002$ 
SNELLING, R.R. 1966. Studies on North American bees of the genus Hylaeus 1. Distribution of the western species of the subgenus Prosopis with descriptions of new forms (Hymenoptera: Colletidae). Los Angeles County Mus. Contrib. Sci. 98: 1-18.

. 1982. The taxonomy of some neotropical Hylaeus and descriptions of New Taxa (Hymenopterra: Colletidae). Bull. So. California Acad. Sci. 81 (1): 1-25.

Toro, H.; Y. Frederick \& A. Henry. 1989. Hylaeinae (Hymenoptera: Colletidae), nueva subfamilia para la fauna chilena. Acta Ent. Chilena 15: 201-204.

Vachal, J. 1901. Contributions Hyménoptériques, II. Ann. Soc. Ent. France 70: 77- 82.

. 1909-1910. Espèces nouvelles ou litigieuses d'Apidae du Haut Bassin du Parana et des Régions contigües et délimitation d'une nouvelle sous-familie Diphaglossinae (Hym.). Rev. d'Ent., Caen, 28: 5-64 (1909); 65-72 (1910).

Recebido em 29.VI.2001; aceito em 22.II.2002.

\section{Índice dos taxa de Hylaeinae neotropicais}

alampes Moure, Hylaeus. 32

albifrontella $($ Cockerell $)=$ gualanicus 49

amazonicus (Gribodo), Hylaeus 32

arenaria $($ Schrottky $)$, praeoc. $=$ psammophi-

lus .41

arsenicus (Vachal), Hylaeus .............................. 33

asper (Vachal), Hylaeus..................................... 33

aspricollis (Vachal), Hylaeus............................ 33

atripes (Vachal), Hylaeus .................................. 33

aztecus (Cresson), Hylaeus (Prosopis) ............. 52

basirufus (Vachal), Hylaeus................................33

benoisti Michener, Hylaeus (Orohylaeus) ........52

bequaerti (Schrottky), Hylaeus .......................... 33

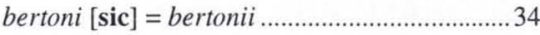

bertonii (Schrottky), Hylaeus .............................. 33

binus (Vachal), Hylaeus .................................... 34

biscutellus (Vachal), Hylaeus............................ 34

bolivianus (Schrottky), Hylaeus .......................... 34

bothros (Schrottky), Hylaeus .............................. 34

brachyceratomerus (Moure), Hylaeus ............... 34

brasiliensis (Schrottky), Hylaeus ........................34

breviradius (Vachal), Hylaeus ............................35

caarendyensis (Schrottky), Hylaeus ..................35

callosa (Friese), praeoc. $=$ callosulus ...............48

callosulus Meade-Waldo, Hylaeus (Hylaeopsis)

cecidonastes Moure, Hylaeus (Hylaeopsis) ..... 48

cockerelli (Schrottky), Hylaeus. coroicensis (Cockerell), Hylaeus ...................... 35

corvicensis $[\mathbf{s i c}]=$ coroicensis .......................... 35

costaricensis (Friese), Hylaeus (Hylaeana) .... 46

cribellatus (Vachal), Hylaeus ............................ 35

cruentus (Vachal), Hylaeus (Gongyloproso-

pis) ........................................................ 45

crustatus (Vachal), Hylaeus.............................. 35

culiciformis (Schrottky), Hylaeus ..................... 36

curtellus Moure, Hylaeus................................... 36

curtulus (Vachal), Hylaeus ............................... 36

cuscoanus (Strand), Hylaeus.............................. 36

dictyotus Snelling, Hylaeus (Hylaeana) .......... 46

dubiosus (Cresson), Hylaeus (Hylaeopsis) ...... 48

exiguus (Schrottky), Hylaeus ………………..... 36

expansus (Vachal), Hylaeus.............................. 36

extrinsecus Snelling, Hylaeus (Hylaeana) ....... 46

femoralis (Schrottky), Hylaeus ......................... 37

fissus (Vachal), Hylaeus ................................... 37

flavohumeralis, (Cockerell), Hylaeus ............... 37

formosus Krombein, Hylaeus ............................ 37

fossisternis $($ Vachal $)=$ bertonii.......................... 34

geminus (Vachal), Hylaeus ............................... 37

genualis (Vachal), Hylaeus ............................... 37

gracillima var. paranensis Schrottky = gracilli-

mus.

gracillimus (Schrottky) Hylaeus (Hylaeopsis). 49

gracillinea $[$ laps.] = gracillimus ....................... 49

grossus (Cresson), Hylaeus (Hylaeopsis) ........ 49 
gualanicus (Cockerell), Hylaeus (Hylaeopsis). 49 guaraniticus (Schrottky), Hylaeus.......................38

hydrophilus (Schrottky), Hylaeus......................38

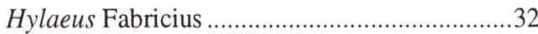

Hylaeus (Cephylaeus) Moure .............................45

Hylaeus (Gongyloprosopis) Snelling ................45

Hylaeus (Hylaeana) Michener ...........................46

Hylaeus (Hylaeopsis) Michener ........................48

Hylaeus (Orohylaeus) Michener .......................52

Hylaeus (Prosopis) Fabricius ............................52

Hylaeus (Spatulariella) Popov ...........................53

iheringi (Schrottky), Hylaeus ..............................38

iridipennis (Schrottky), Hylaeus ........................38

itapuensis (Schrottky), Hylaeus...........................38

joergenseni (Schrottky), Hylaeus .......................39

knabi (Cockerell), Hylaeus (Hylaeana)............46

larocai Moure, Hylaeus (Cephylaeus) ..............45

limbifrons (Cresson), Hylaeus...........................39

longicornis $($ Schrottky), praeoc. $=$ seabrai ......43

lumbellus (Vachal), Hylaeus ..............................39

lychnis (Vachal), Hylaeus..................................39

maculata $($ Friese $)=$ grossus ..............................49

maculipennis (Smith), Hylaeus (Hylaeopsis) ...50

maculosus (Friese), Hylaeus (Hylaeopsis) ........50

mapirensis (Schrottky), Hylaeus .......................39

mapiriensis $[\mathbf{s i c}]=$ mapirensis $\ldots \ldots \ldots \ldots \ldots \ldots \ldots \ldots \ldots . . . . . . . .39$

merelus $[\mathbf{s i c}]=$ puerullus ..................................41

mexicanus (Cresson), Hylaeus (Hylaeopsis) ....50

monacha $($ Warncke $)=$ callosulus .......................48

nasutus (Vachal), Hylaeus.................................39

nigripennis (Vachal), Hylaeus ...........................39

opaciventris (Friese), Hylaeus (Hylaeopsis) ....50

opacus (Schrottky), praeoc.= joergenseni ........39

orbicus (Vachal), Hylaeus (Gongyloprosopis).45

palmaris (Vachal), Hylaeus.

panamensis Michener, Hylaeus (Hylaeana) ....46

pannosus (Vachal), Hylaeus.

paradoxus (Schrottky), Hylaeus ........................40

paraguayensis (Schrottky), Hylaeus .................40

paulistanus (Schrottky), Hylaeus .......................40

peruvianus (Schrottky), Hylaeus ........................40

petroselini (Schrottky), Hylaeus .........................41 phaeoscapus Snelling, Hylaeus (Hylaeana) ....47

phaeoscopus $[\mathbf{s i c}]=$ phaeoscapus .....................47

polybioides (Schrottky), Hylaeus .......................4 41

polybiaeformis (Schrottky), Hylaeus ................ 41

preposterosus Snelling, Hylaeus (Gongyloproso-

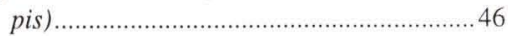

psammophilus (Schrottky), Hylaeus .................41

puerulus (Vachal), Hylaeus............................. 41

punctatus (Brullé), Hylaeus (Spatulariella) ..... 53

quadratifer (Cockerell), Hylaeus (Hylaeana) ..47

rawi Snelling, Hylaeus (Hylaeana) ...................47

recisus (Vachal), Hylaeus .................................. 41

rivalis (Schrottky), Hylaeus .............................. 41

royesi Raw, Hylaeus (Hylaeana) ...................... 47

ruficollis $($ Friese $)=$ gualanicus ........................ 49

rufoclypeatus (Friese), Hylaeus (Hylaeana) ....47

rufulus (Friese), Hylaeus ................................... 42

rugosus (Smith), Hylaeus.................................. 42

saltensis (Friese), Hylaeus ............................... 42

saltensis var. rufifrons (Friese) $=$ saltensis ...... 42

scabra $($ Vachal $)$ praeoc. $=$ vachali ..................44

scrobicauda (Vachal), Hylaeus ........................ 42

scrupeus (Vachal), Hylaeus .............................. 42

sculptilis (Schrottky), Hylaeus ........................... 42

seabrai Urban \& Moure, Hylaeus ...................... 43

stenops (Schrottky), Hylaeus ............................ 43

stilbaspis (Vachal), Hylaeus............................... 43

subgriseus (Cockerell), Hylaeus (Hylaeopsis). 50

titanius (Friese), Hylaeus (Hylaeopsis) .............51

transversus (Vachal), Hylaeus ......................... 43

transvittatus (Cockerell), Hylaeus (Prosopis).. 52

triangulum (Fabricius), Hylaeus ...................... 43

trepanda $($ Smith $)=$ vigilans .............................51

tricolor (Schrottky), Hylaeus (Hylaeopsis) ......51

tristis $($ Schrottky) praeoc. $=$ yaguarae $\ldots \ldots \ldots \ldots . . . .45$

tristissimus Meade-Waldo = yaguarae .............45

trisulcus (Vachal), Hylaeus ............................... 44

trivittatus (Friese), Hylaeus (Hylaeana) ...........48

trivittatus var. maculosus (Friese) = maculosus...

ubertus (Vachal), Hylaeus................................. 44

vachali Meade-Waldo, Hylaeus ......................... 44 
vachalianus Moure, Hylaeus............................. 44

variolosus (Smith), Hylaeus ............................... 44

vigilans (Smith), Hylaeus (Hylaeopsis) ............51

villosellus Moure, Hylaeus ................................ 44

xanthocephalus (Schrottky), Hylaeus ................44

yaguarae (Schrottky), Hylaeus .45

zamoranicus (Cockerell), Hylaeus (Hylaeop-

sis)

\section{Índice das plantas}

Alyssum saxatile L. (Brassicaceae) .53

Aloysium citriodora Orteg. ex Pers. [= Lippia citriodora] .53

bambus [Poaceae] .51

Cichorium intybus L. [Asteraceae] .53

Compositae $=$ Asteraceae 47

Dicraurus Hook. f. [Amaranthaceae]. .47

Elvetia tinifolia $\mathrm{L}$ . .51

Hyptis emoryi Torreyi [Lamiaceae] .47

Iresine diffusa H. \& B. ex Willd. [Amaranthaceae]

Iresine paniculata L. [= Iresine diffusa]

Jussiacea sericea Camb.

[= Ludwigia sericea $]$ 42

Lantana L. [Verbenaceae] 53

Lippia citriodora H. B. K. [Verbenaceae] .......53 Ludwigia sericea (Camb.) Hara [Onagraceae]. 42 Mikania micracantha Kunth. [Asteraceae] ......47 Schinus engleri $\mathrm{F}$. A. Barkley [Anacardiaceae]..
Petroselinum crispum (Miller) A. W. Hill [Apiaceae].... 35, 36, 37, 38, 41, 42, 43, 44, 45, 51

Petroselinum sativum Hoff. [= Petroselinum crispum]

Verbesina encelioides Benth et Hook. [Asteraceae]

\section{Outros Insecta}

Apis mellifera L., 1758 [Hymenoptera, Apidae]..

Bothranthidium Moure, 1947 [Hymenoptera, Megachilidae] ............................................ 43

Cecidoseidae, Lepidoptera .................................. 48

Eucecidoses minutans Brèthes, 1916 (Cecidoseidae) .48

Gorytes Latreille, 1804 (Hymenoptera, Crabronidae)

Manuelia Vachal, 1905 [Hymenoptera, Anthophoridae] .53

Mariposas [Lepidoptera] .53

Mischocyttarus cassununga (R. von Ihering, 1903) [Hymenoptera, Vespidae]

Polybia scutellaris (White, 1841) [Hymenoptera, Vespidae]

Sirfídeos [Diptera, Syrphidae] .53

Sphecodes Latreille, 1804 [Hymenoptera, Halictidae] 43

Trypoxylum fabricator Smith, 1873 [Hymenoptera, Crabronidae]. 51 